\title{
Energy Evaluation Model for an Improved Centralized Clustering Hierarchical Algorithm in WSN
}

\author{
Mian Ahmad Jan, Priyadarsi Nanda, Xiangjian He \\ Centre for Innovation in IT Services and Applications (iNEXT) \\ University of Technology, Sydney \\ Sydney, Australia \\ Mian.A.Jan@student.uts.edu.au \\ Priyadarsi.Nanda@uts.edu.au \\ Xiangjian.He@uts.edu.au
}

\begin{abstract}
Wireless Sensor Networks (WSN) consists of battery-powered sensor nodes which collect data and route the data to the Base Station. Centralized Cluster-based routing protocols efficiently utilize limited energy of the nodes by selecting Cluster Heads (CHs) in each round. Selection of $\mathrm{CHs}$ and Cluster formation is performed by the Base Station. In each round, nodes transmit their location information and their residual energy to the Base Station. This operation is a considerable burden on these resource hungry sensor nodes. In this paper we propose a scheme whereby a small number of High-Energy nodes gather location information and residual energy status of the sensing nodes and transmit to the Base Station. This scheme eliminates $\mathrm{CH}$ advertisement phase in order to conserve energy. Based on the energy consumption by various types of nodes, we have derived an energy model for our algorithm which depicts the total energy consumption in the network.
\end{abstract}

Keywords: Wireless Sensor Network, LEACH, Cluster Head, Base Station.

\section{INTRODUCTION}

Micro-Electro-Mechanical Systems (MEMS) technology has seen phenomenal growth in recent years which has enabled the deployment of small, battery powered sensor nodes. These tiny sensor nodes have brought a revolution in the world of Wireless Communication System [1]. Sensor nodes are constraint with resources like energy, storage, processing capabilities and available bandwidth. Sensor nodes collaborate with each other to form Wireless Sensor Network (WSN) which outperforms many existing Wired and Wireless technologies in terms of their use. These networks are deployed in environments which cannot be monitored with traditional networks. They have numerous applications like volcanic monitoring, battlefield surveillance, home appliances, industrial monitoring, agriculture, health and many more. These networks possess some unique characteristics like self-healing, self-organizing and fault tolerance. WSN is considered as a next wave of computing. WSN market is expected to grow from its current half a billion dollars to around two billion dollars by 2021 [2]. Sensor nodes are randomly deployed in most applications and left unattended. Hence it becomes necessary that the energy of the nodes should be utilized efficiently to extend the lifetime of the network. Energy-efficient routes need to be setup and the data should be reliably transferred from the nodes to the Base Station [3]. If all these nodes route their data directly to the Base Station, large amount of energy will be consumed due to long range communication. Hence nodes are grouped together to form Clusters. In Clustering protocols, one node is elected as Cluster Head (CH) which gather, aggregate and route the data to the Base Station [4] These CHs can either transmit the data directly to the Base Station or route their data to the upper level $\mathrm{CHs}$ and hence form a hierarchy of Clusters to reach the Base Station.

In existing Clustering Hierarchical protocols, the $\mathrm{CH}$ is either selected randomly or this task being performed by Base Station. Base Station uses the residual energy and location information of the nodes as selection criteria. However in this scheme, the nodes have to transmit their location information and energy status at the start of each round to the Base Station. Although, this mechanism results in an optimal number of CHs in each round but it consumes a considerable amount of energy.

In this paper, we have proposed a centralized solution for $\mathrm{CH}$ selection. In our approach, $\mathrm{CH}$ selection and Cluster formation are performed by the Base Station. Here, we have considered five percent of the nodes as High-Energy nodes in addition to sensing nodes in any sensor network. As per the above statistics, a network of hundred sensing nodes would require an additional five High-Energy nodes and a network of two hundred sensing nodes would require an additional ten such nodes. The number of such nodes in any network depends on 
its scalability. These High-Energy nodes transmit the location and residual energy of the sensing nodes to the Base Station in each round. They are located in the sensor field in order to enable the sensing nodes to minimize their energy consumption during communication. Once they transmit the sensing node's location and energy information to the Base Station, they go to sleep mode till the beginning of next round. The Base Station selects optimal number of CHs.

Also, our proposed algorithm eliminates "CH-Advertisement". $\mathrm{CHs}$ are no longer required to advertise themselves to their respective Cluster members. This approach enables the $\mathrm{CH}$ nodes to conserve their energy. We have calculated the energy consumption by different nodes in the network and formulated a new energy model. Our model then depicts the total amount of energy consumed in the network.

This paper is organized into six sections. In section II we presented related works and refined our objectives for a new Centralized $\mathrm{CH}$ selection and Cluster formation approach. In Section III we presented radio model for sensor nodes of our algorithm. In Section IV we have described the network architecture model and the operations performed by the Base Station for Cluster formation and $\mathrm{CH}$ selection. In Section V, we have derived an energy model for our algorithm. We concluded our work in section VI with future directions.

\section{RELATED WORKS}

A Centralized Low Energy Adaptive Clustering Hierarchy (LEACH-C) protocol was presented in [5]. In this protocol, the Base Station elects the optimal number of Clusters and CHs using simulated annealing algorithm [6]. At the start of each round, the nodes send their remaining energy levels and location information to the Base Station which in turn calculates the average of these residual energy levels. All those nodes whose energy levels are greater than average value are elected as $\mathrm{CHs}$. Practically this approach cannot guarantee the optimal number of CHs. It is highly probable that in each round, there will be a considerable number of $\mathrm{CHs}$ exceeding the optimal value. Also, this protocol lacks the detail mechanism of its operation. This protocol is an improvement over LEACH [7], where each node autonomously elects itself as CH. LEACH results in poor Cluster formation and nodes with very low energy are frequently selected as $\mathrm{CHs}$.

Base-Station Controlled Dynamic Clustering Protocol (BCDCP) was proposed in [8]. BCDCP uses the same centralized approach for Cluster formation as of LEACH-C. Additionally, the Base Station maintains a set $\mathrm{S}$, which contains all those nodes whose energy levels are greater than average energy value. The task of the Base Station is to elect an optimal number of Cluster Heads $\left(\mathrm{N}_{\mathbf{C H}}\right)$ from $\mathrm{S}$ and arrange all the remaining nodes of the network around them. BCDCP implements an iterative Cluster splitting algorithm which splits the sensor network into two sub-Clusters and proceed further until sufficient numbers of small Clusters are formed. The number of sub-Clusters depends on the number of $\mathrm{N}_{\mathbf{C H}}$ nodes. BCDCP uses balanced Clustering technique [9] to establish Clusters having approximately equal number of nodes. This protocol consumes a considerable amount of time in Cluster formation and $\mathrm{CHs}$ selection and hence does not suit time critical applications.

Base-Station Initiated Dynamic Routing Protocol (BIDRP) is proposed in [10]. All the above protocols emphasis on the homogenous sensor networks where all the nodes have the same initial energy. BIDRP is specifically used for heterogeneous sensor networks where some nodes have higher energy in the network. These higher energy nodes are always elected as $\mathrm{CHs}$ in each round. The Base Station broadcasts a packet which is specifically meant for these higher energy $\mathrm{CH}$ nodes which in turn calculate their distance from the Base Station based on the Received Signal Strength (RSS) of the packet. This enables the CHs to adjust their transmission power in order to reach Base Station in one hop. Next, these CHs transmit the same packet to the $\mathrm{CHs}$ which are one level higher. They also perform the same function and adjust their transmission power and this process continues till the highest level of CHs. Base Station is at Level 0, the CHs which are one hop away from Base Station are at Level 1 and so on. Cluster Heads aggregate their data from their respective Clustering nodes and deliver it to the $\mathrm{CHs}$ which are one-hop up the tree. Data always flow from higher levels to lower level until it is delivered at the Base Station. BIDRP lacks the concept of CH rotation. Same higher energy nodes are used as $\mathrm{CHs}$ in each round, ultimately their energy will drain after some time. For video streaming and multimedia applications, this protocol does not produce the best results as these applications have considerable amount of data and require constant availability of services. BIDRP does not provide an alternative solution in situations where these higher energy $\mathrm{CH}$ nodes die. Clustering Protocol with Mode Selection (CPMS) is proposed in [11]. This protocol is based on [8] for $\mathrm{CH}$ selection and on [10] for levels formation. CPMS improve the selection of Leader node based on Energy-Distance (E-D) ratio. Residual energy of each $\mathrm{CH}$ node is divided by their respective levels to obtain E-D ratio. The one whose E-D ratio is highest is elected as the Leader node which will gather the data from all $\mathrm{CHs}$ and transmit to Base Station. The drawback of this 
approach is that $\mathrm{CHs}$ does not rotate like [10] and $\mathrm{CH}$ nodes far away from Base Station have less chance to be elected as Leader nodes.

All these protocols have one major drawback in common: all the nodes transmit their residual energy status to the Base Station at the start of each round which is a considerable burden on these energy-constraint sensor nodes. Alternatively, there should be additional solution to broadcast energy status of sensor nodes in each round which can minimize the energy consumption. Our algorithm is based on the latter, where a small number of High-Energy nodes perform transmission of location and residual energy information of the sensing nodes to the base station. Base Station evaluates the energy level of all the nodes and calculates an average threshold value. Nodes are selected as Cluster Heads based on their energy values. If their energy level is greater than threshold value, they are eligible to be elected as Cluster Heads. More than one node in the same cluster cannot be elected as Cluster Head. Nodes having energy values greater than average threshold energy level are elected as Candidate nodes. The choice of Cluster Head node among the Candidate nodes depends on various factors like the specific number of Candidate nodes in a particular Cluster, Candidate node elected as Cluster Head in previous round, its present energy status.

\section{SENSOR NODE RADIO MODEL}

The radio model used by the sensor nodes in our network is shown in Figure 1 and is based on [12].

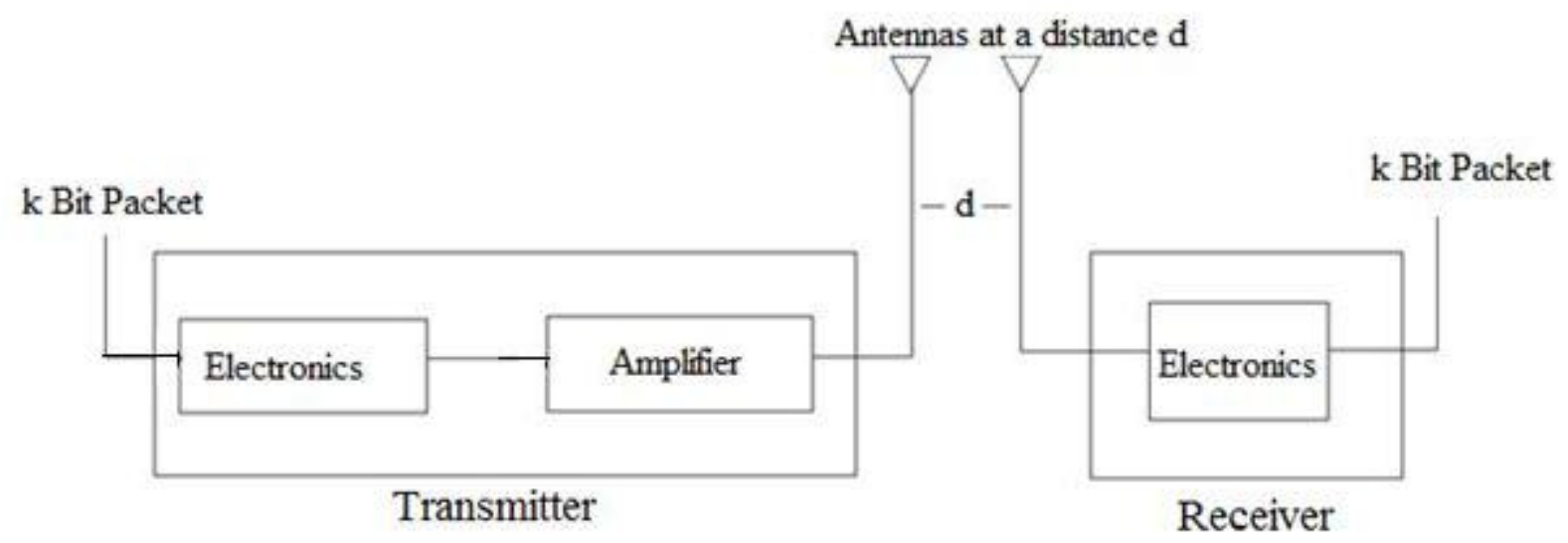

Fig.1. Sensor Node Radio Model

In Figure $1, \mathrm{k}$ is the length of message and $\mathrm{d}$ is the distance between the receiver and transmitter nodes. $\mathrm{E}_{\mathrm{Tx}}$ and $\mathrm{E}_{\mathrm{Rx}}$ are the energy consumed by the transmitter and receiver nodes respectively. $\mathrm{E}_{\mathrm{Tx}}$ is equal to the sum of energy dissipated by the Transmitter's electronic $\left(E_{e l e c}\right)$ and its amplifier $\left(\varepsilon_{\mathrm{amp}}\right)$ while $\mathrm{E}_{\mathrm{Rx}}$ is equal to the energy dissipated by the receiver's electronics $\left(E_{\mathrm{elec}}\right)$. Their values are given below in the equation 1 and 2 respectively [12].

$$
\begin{gathered}
E_{T X}(k, d)=E_{\text {elec }} k+\varepsilon_{\text {amp }} k d^{n} \\
E_{R x}(k)=E_{\text {elec }} k
\end{gathered}
$$

The amount of energy consumed by the transmitter's amplifier depends on the distance (d) between the transmitter and receiver node. If the distance $(d)$ is less than crossover distance $\left(\mathrm{d}_{\text {crossover }}\right)$, then path loss $(n)$ is modeled by free space [12]. FS-amp stands for energy consumed by amplifier in free space. The amount of energy consumed by the transmitter in this case is given by equation 3 :

$$
E_{T X}(k, d)=E_{\text {elec }}(k)+\varepsilon_{F S-a m p}(k) d^{2}
$$

If the distance $\mathrm{d}$ is greater than or equal to $\mathrm{d}_{\text {crossover, }}$ then Two-Ray model (multipath) is used. Here equation 1 is modified as following: 


$$
E_{T x}(k, d)=E_{\text {elec }}(k)+\varepsilon_{T w o-R a y-a m p}(k) d^{4}
$$

In free space, there is a line of sight connection between transmitter and receiver while in Two-Ray ground propagation model, signal travel through multi paths due to deflection through obstacles. The crossover distance is given in equation 5 :

$$
d_{\text {crossover }}=4 \pi \sqrt{L h_{t} h_{r}} / \lambda
$$

Here $h_{t}$ and $h_{r}$ are the height of sender and receiver antennas respectively above the ground, $L$ is the system loss and $\lambda$ is the signal wavelength. Crossover distance is $87 \mathrm{~m} \mathrm{[12].}$

Also we calculated the amount of energy consumed by a sensor node in sensing the environment and is equal to

$$
E_{\text {Sense }}=\alpha . I / \text { UnitTime }
$$

Where $\alpha$ is the amount of energy consumed by a sensor node to sense a single bit and I is the total number of bits in a message being sensed.

\section{NETWORK ARCHITECTURE}

The network architecture of our proposed model is based on the following assumptions:

- Base Station is located far away from sensor field and is immobile.

- Sensor nodes are energy-constrained and have the same initial energy.

- Communication channels are symmetric in nature i.e.: energy consumed in transmission on a particular channel is equal to energy consumed for reception on it.

- Sensor nodes have the capabilities to adjust their transmission power.

- Sensor nodes sense the environment at a fixed rate and always have data to send.

Additionally, our network consists of a small percentage of High Energy nodes (other than sensor nodes) which have higher energy than the sensor nodes deployed. Also, optimal number of CHs in each round is assumed around five percent of the sensor nodes. The reason we have chosen five percent of high energy nodes to balance between computational complexity to run our algorithm and lower data aggregation ratio against high energy efficiency and transmission distance.

Our proposed algorithm is based on centralized approach: Cluster formation and CHs selection is performed by the Base Station. We believe our approach is unique because it uses a hybrid methodology: For Cluster formation and $\mathrm{CHs}$ selection, it is homogenous because only sensing nodes can be elected as $\mathrm{CHs}$ and belongs to any of the Clusters formed by the BS. All these sensing nodes have the same initial energy at the time of deployment. For transmission of energy status of nodes and location information, it is heterogeneous because such information is only transmitted by High-Energy nodes which have higher energy than sensing nodes. HighEnergy nodes do not perform any sensing and only perform transmission of location information and residual energy to BS at the start of each round.

In this section the flowchart for our proposed algorithm is presented. The flowchart illustrates the Setup phase of our algorithm followed by the network operation. Evaluation of residual energy levels by the Base Station is also presented here which explain the queuing mechanism being deployed by the Base Station and the selection criteria for a node to be elected as Cluster Head. The BS maintains a single queue which contains the location information and residual energy information of each node. Base Station chooses $\mathrm{CHs}$ from the queue by evaluating each node's residual energy against average energy threshold value. Location information of each sensing node (other than High-Energy nodes) is also maintained in the queue. Once BS elects the CHs nodes, location information of each node is used to transmit this information to each individual node. High-Energy nodes do not participate in $\mathrm{CH}$ election or Cluster formation. The flowchart, network operation and evaluation procedure by the Base Station in our proposed approach are presented in this section. 


\subsection{FLOWCHART OF THE ALGORITHM}

In this sub-section, a detail flowchart for our algorithm is presented. In the flowchart

- L stands for Location

- RE stands for Residual Energy of Nodes

- HEN stands for High-Energy Nodes

- CN stands for Candidate Node(s)

- A is connector

The algorithm describing our scheme is presented through the flow chart in Figure 2.

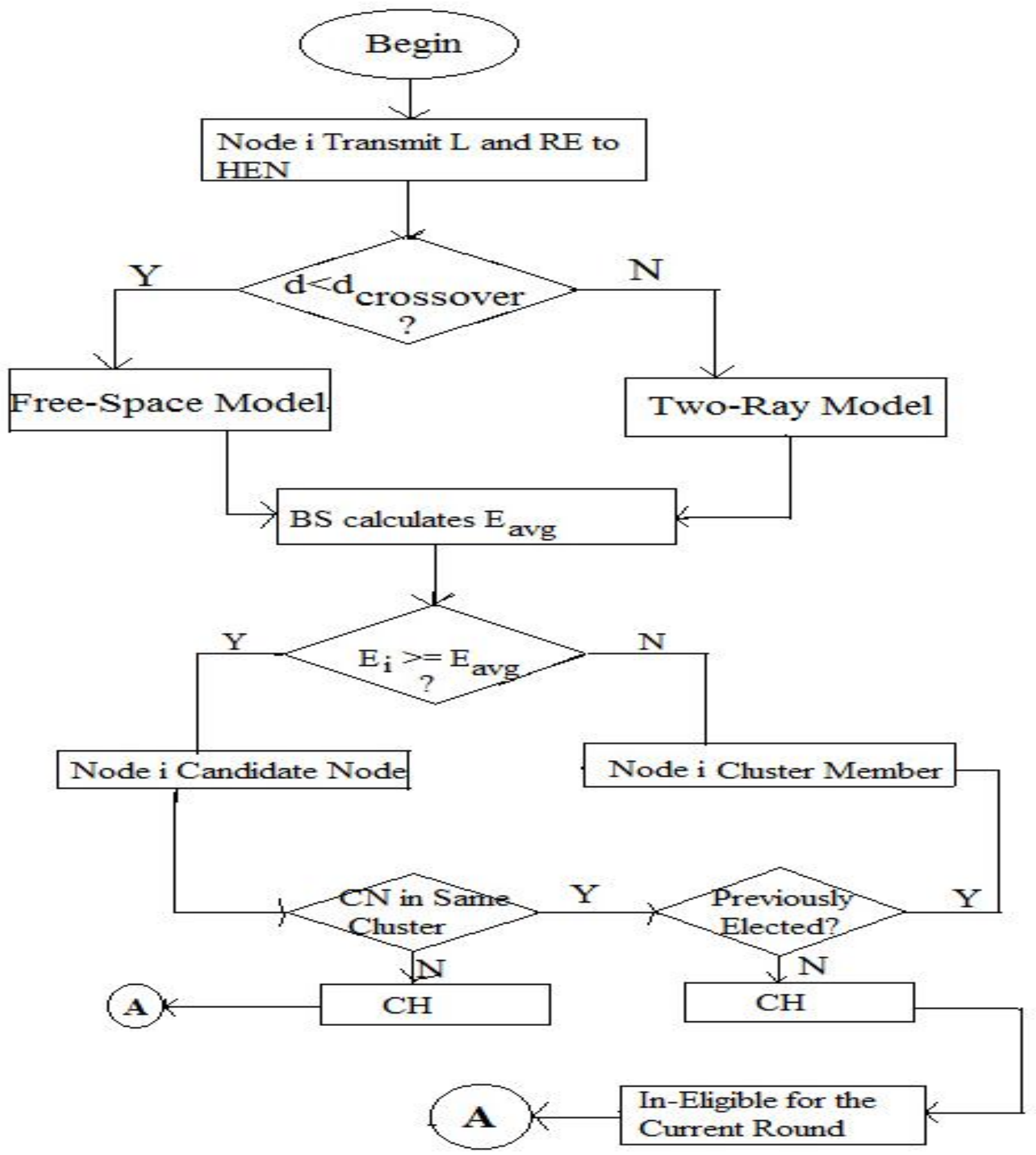




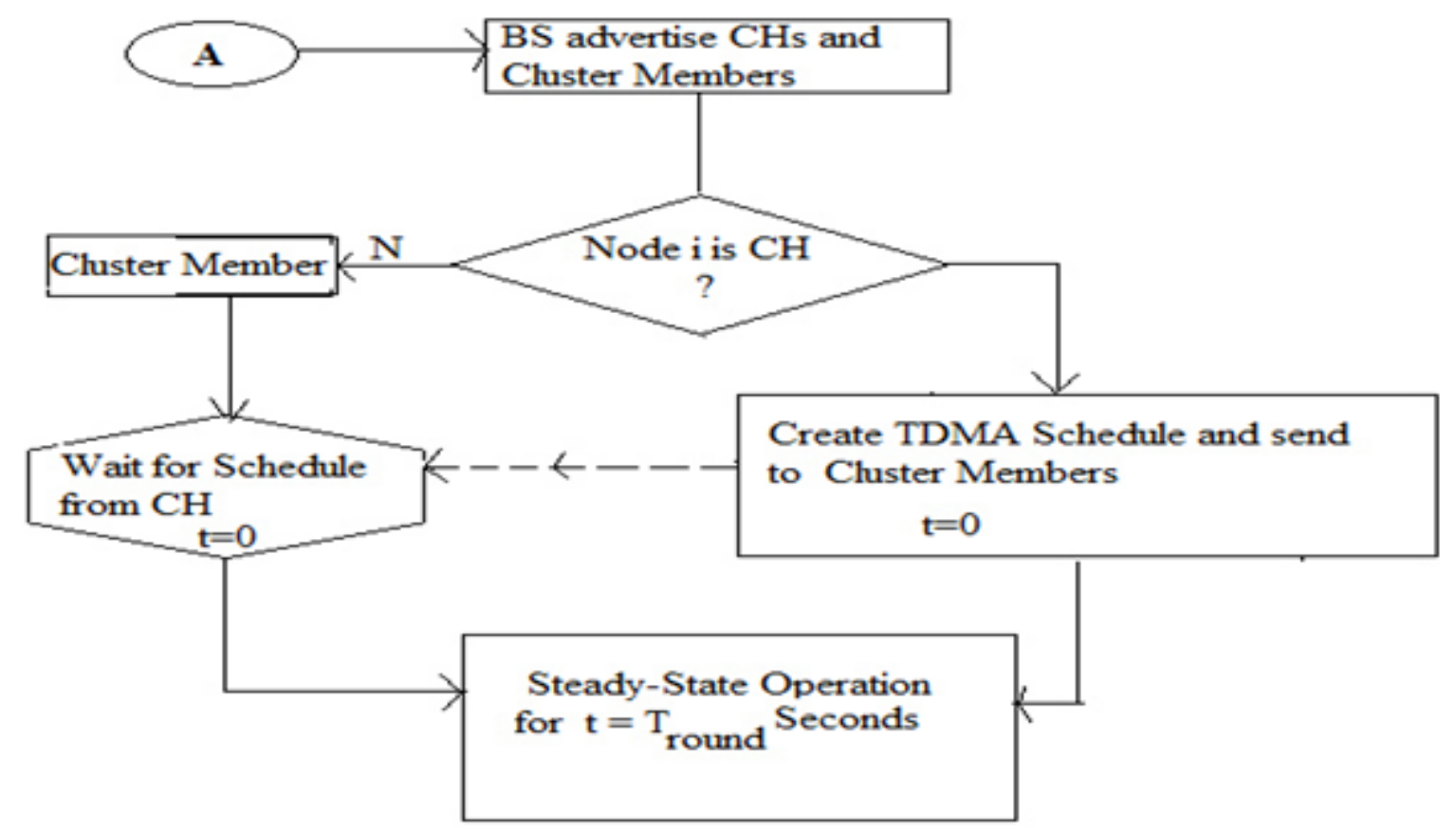

Fig.2. Flowchart of the Proposed Algorithm

\subsection{NETWORK OPERATION}

Our proposed algorithm operates in two phases: Setup phase and Steady-State phase. Setup phase consists of the following sub-phases: Status, Cluster formation, Cluster Head selection and Schedule creation.

In Status sub-phase, all the nodes transmit their location information and residual energy at the start of each round to the High-Energy nodes instead of Base Station. These High-Energy nodes are uniformly deployed in the network as shown in Figure 3. High-Energy nodes can be deployed outside the sensor field. However in that case, the sensor nodes have to transmit over a longer range to reach them. Also, at later stages when the nodes are low on energy, they cannot afford to transmit over longer range. In-network deployment High-Energy nodes enhance the lifetime of the network. These nodes are used only to transmit Status message containing residual energy and location information of nodes to the Base Station.

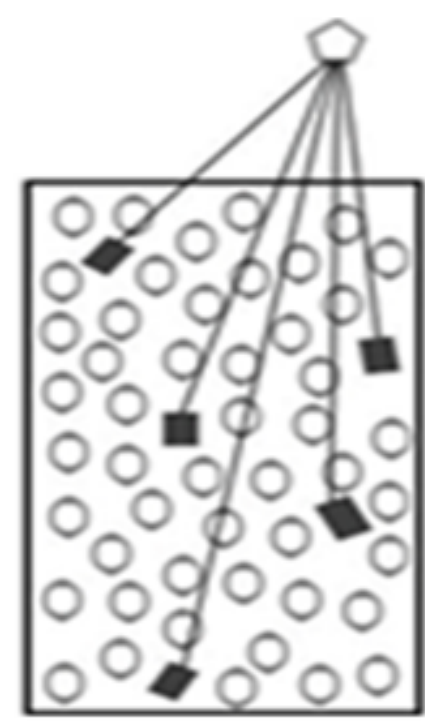

Base Station

High-Energy Nodes

Sensor Node

Fig.3. Operation of High-Energy Nodes 
The Status message contains Source ID, destination ID and residual energy of the node as shown in Figure 4. Destination ID is always the ID of one of the High-Energy nodes. Source ID is of the source node which transmits status message. Residual energy field is variable in length.

\begin{tabular}{|l|l|l|}
\hline Source ID & Destination ID & Residual Energy \\
\hline
\end{tabular}

Fig.4. Frame Format of Status Message

Once the High-Energy nodes deliver the Status message to the Base Station, they go to sleep mode until the start of next round. The Base Station maintains a queue containing all the nodes having their location information and energy status (Figure 5a). In our network, there are 100 nodes other than the High-Energy nodes. HighEnergy nodes in our proposed algorithm are only five percent in addition to the normal sensing node but it can be increased up to ten percent. The reason we have chosen five percent of such nodes is because greater the number of High-Energy nodes means higher the network cost as these nodes are relatively expensive than normal sensing nodes. The Base Station calculates the average $\left(\mathrm{E}_{\mathrm{avg}}\right)$ of all the energy levels of the nodes using the formula

Where;

$$
E_{\text {avg }}=\sum_{i=1}^{i=100}\left(E_{i} / N\right)
$$

$\mathrm{E}_{\mathrm{i}}$ is the energy of node $\mathrm{i}, \mathrm{N}$ is the total number of sensing nodes in the network and is equal to 100.

Any node whose energy level is greater than $\mathrm{E}_{\text {avg }}$ is eligible to be elected as a $\mathrm{CH}$. It is highly probable that there will be a large number of candidate nodes for $\mathrm{CHs}$ (Figure 5c). Base Station evaluates such candidate nodes and selects an optimal number of nodes as CHs. In our network, optimal value is five (Figure 5d). All these various operations are shown below in Figure 5.
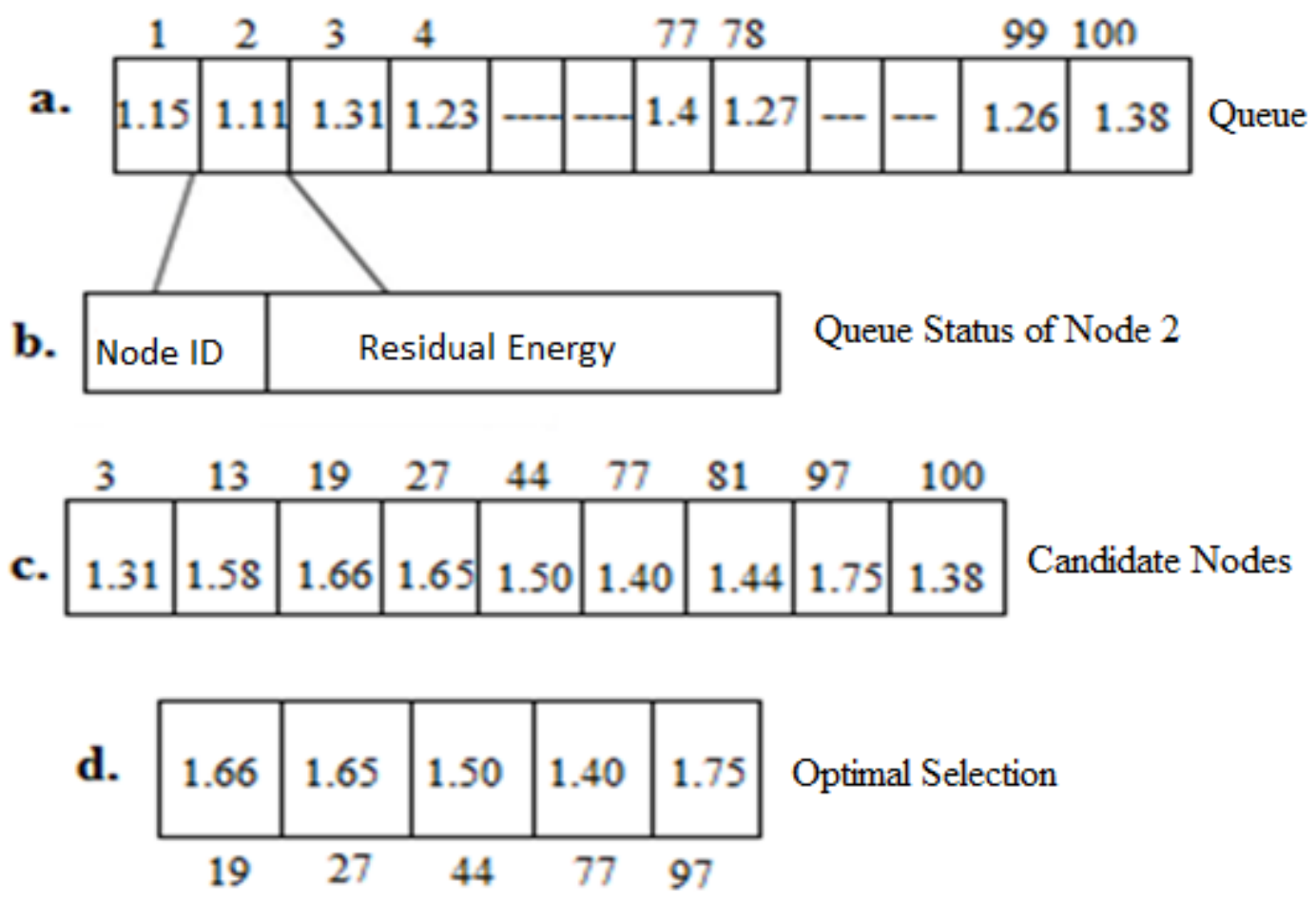

Fig.5. Cluster Head Selection Procedure by Base Station 


\subsection{EVALUATION OF RESIDUAL ENERGY BY BASE STATION}

The Base Station evaluates energy levels of the nodes and results in nine nodes eligible as $\mathrm{CH}$ candidates as shown in Figure 5c. Candidate nodes are those having residual energy greater than average value. As the optimal number of $\mathrm{CHs}$ in each round are five so the Base Station further evaluates these nine nodes and finalize five of them. Like BCDCP, CPMS and BIDRP, our algorithm is also based on balanced Clustering Technique [9]. However, the procedure for evaluation of Candidate nodes is totally different. In Equation 5, the average threshold energy value for a sensor node to be nominated as a Candidate node for $\mathrm{CH}$ is 1.30 Joule. This value is calculated by the Base Station by taking the average of all energy values of sensing nodes. However, it is important to note that the average threshold value for a $\mathrm{CH}$ Candidate node changes in each round. In the beginning the sensor nodes have higher energy, so the average threshold energy value is higher. In the latter rounds when the energy level of the nodes reduces due to extensive network operations, the average threshold energy values also reduces.

Among the Candidate nodes, 3, 13 and 19 lies in the same Cluster. Nodes 81, 97 and 100 also lie in the same Cluster (Figure 6). Only one node in each Cluster has to be elected as CH by the Base Station. Since nodes 19 and 97 have higher energy in their respective Clusters so they are chosen as $\mathrm{CH}$ nodes. Selection of Nodes in a particular Cluster depends on their residual energy and whether it has been elected as $\mathrm{CH}$ in previous rounds or not. If in case, node 19 and 97 are elected in previous rounds, they become ineligible to become CHs in this round. In this figure, Candidate nodes in Cluster 1 and 5 are represented by dark circles with numerical numbers. Bold numeric digit in each cluster represents their respective Cluster number. All these tasks are performed by the Base Station which has sufficient energy to perform this complex computation task.

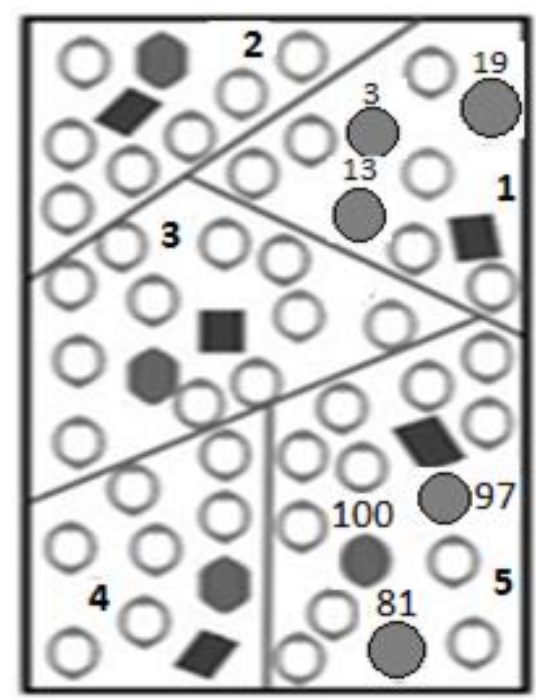

\section{Candidate Nodes in Cluster 1 and}

5 Competing with each others

Fig.6. Candidate Nodes

The term "Cluster" used in Figure 6 and preceding figures are from the prospective of Base Station. At the time of evaluation by the Base Station, there are neither $\mathrm{CHs}$ nor Clusters. Once the Base Station evaluates the energy levels, then CHs are selected and Clusters are formed. In the beginning, all these nodes lie in the "Region" of sensor network. So Region is the most suitable word to be mentioned in place of Cluster in these Figures. However, for simplicity we have used the word Cluster.

Once CHs are selected, the Base Station transmits a message to each node about the notification of their respective CHs. This message contains each node ID and their respective Cluster Head ID. The reason we have chosen this method is because the Base Station already has location information of all the nodes. So it is the job of Base Station to assign Cluster Head to each node. CHs will conserve lot of energy as they are no longer required to broadcast "CH-advertisement" messages to their respective Cluster members. Once the nodes receive this message from the Base Station, they associate themselves with their respective CHs (being assigned by the Base Station). During all these operations by the Base Station, sensor nodes and the High-Energy nodes remain in sleep mode in order to conserve their energy. The $\mathrm{CHs}$ create Time Division Multiple Access (TDMA) schedule for their respective Cluster members to transmit data during their respective slots. 
Once Setup phase is completed, Steady-State phase starts. In this phase, the CHs gather data from their respective Clustering nodes and transmit to one of the $\mathrm{CH}$ node which is elected by the Base Station as the Leader node. Unlike other protocols, our Leader node changes in each round and is always elected by the Base Station based on the residual energy of $\mathrm{CH}$ nodes. This balances the energy load uniformly among all the Leader nodes.

\section{ENERGY MODEL}

Based on [12], we have calculated the energy consumed by $\mathrm{CH}$ nodes, Leader node (LN), High-Energy nodes (HENs) and normal sensing nodes (SN) of Figure 7. As previously said, our network has 100 nodes $(\mathrm{N}=100)$ (other than High-Energy nodes) in a 100m $x 100 \mathrm{~m}$ sensor field. The base station is located $90 \mathrm{~m}$ from its nearest sensor neighbor. The energy consumed by the $\mathrm{CH}$ nodes is

$$
E_{C H}=m E_{\text {elec }}(N / k)+m E_{D A}((N / k)-1)+m_{\varepsilon_{F S-a m p}} d^{2} \text { to-LN }
$$

Unlike LEACH-C and its variants, the $\mathrm{CHs}$ in our network transmit the data to another $\mathrm{CH}$ having large energy (Leader node) instead of BS. Since Leader node is located in the field of above dimensions so free space model is used. $\mathrm{k}$ is the optimal number of Clusters (here $\mathrm{k}=5$ ), $\mathrm{E}_{\mathrm{DA}}$ is the energy consumed in data aggregation while $\mathrm{m}$ is the message being transmitted by each Clustering node. Next, each $\mathrm{CH}$ transmits the aggregated data to the Leader node (LN).

The energy consumption of the High-Energy node (HEN) is calculated as:

$$
E_{\text {HEN }}=m E_{\text {elec }}+m \varepsilon_{\text {Two-Ray-amp }} d^{4}{ }_{\text {to-BS }} \text { (9) }
$$

Here, $\mathrm{m}$ is the message sent by each node containing its location information and residual energy during Status sub-phase. $\mathrm{R}$ is the number of nodes. Here $\mathrm{R}$ is variable as the numbers of nodes, which transmit their location and energy information to HEN nodes varies. As HEN nodes transmit this information directly to Base Station which is far away from sensor field, so Two-Ray ground propagation model is used $\left(\mathrm{d}>\mathrm{d}_{\text {crossover }}\right)$. If the HEN nodes are near the Base Station, then Free Space model is used. In our algorithm, Two-Ray ground propagation model will always be used due to our network specification (Distance of Base Station from Sensor field).

The energy consumed by the Leader node is

$$
\begin{gathered}
E_{L N}=m E_{\text {elec }}(N / k)+m E_{D A}((N / k)-1)+n E_{D A}\left(\sum_{i=1}^{l=k-1} C H\right)+ \\
m \varepsilon_{T w o-R a y-a m p} d_{\text {to-BS }}^{4} ; d>d_{\text {crossover }}
\end{gathered}
$$

As the sensor nodes are at a distance greater than crossover from Base Station, so Two-Ray ground propagation model is used to model the energy consumption of the Leader node.

In equation 10, the Leader node collects data from its Clustering members as well (since Leader node is one of the $\mathrm{CH}$ nodes having high energy) and also it gathers data from other $\mathrm{CH}$ nodes. In equation $10, \mathrm{n}$ is the number of messages transmitted by the $\mathrm{CH}$ nodes and k-1 is the number of optimal Clusters from which the data is send. If $\mathrm{k}$ is 5 , this means four $\mathrm{CHs}$ will transmit data to the Leader node.

The energy consumed by any sensing node during Status sub-phase is given by:

$$
E_{\text {Sensing-Node }}=m E_{\text {elec }}+m \varepsilon_{F S-a m p} d^{2}{ }_{\text {to-HEN }}
$$

Sensing node can be any node in the network other than HEN nodes because during Status sub-phase there is neither $\mathrm{CH}$ nor Leader node. Once Clusters are formed and the Clustering nodes start transmitting their data to the $\mathrm{CH}$, their energy consumption is calculated as:

$$
E_{\text {Non-CH }}=m E_{\text {elec }}+m \varepsilon_{F S-a m p} d_{\text {to-CH }}^{2}
$$


Finally, the total energy consumed in the network is equal to the sum of energy consumed by all the nodes of the network and is given in Equation 13.

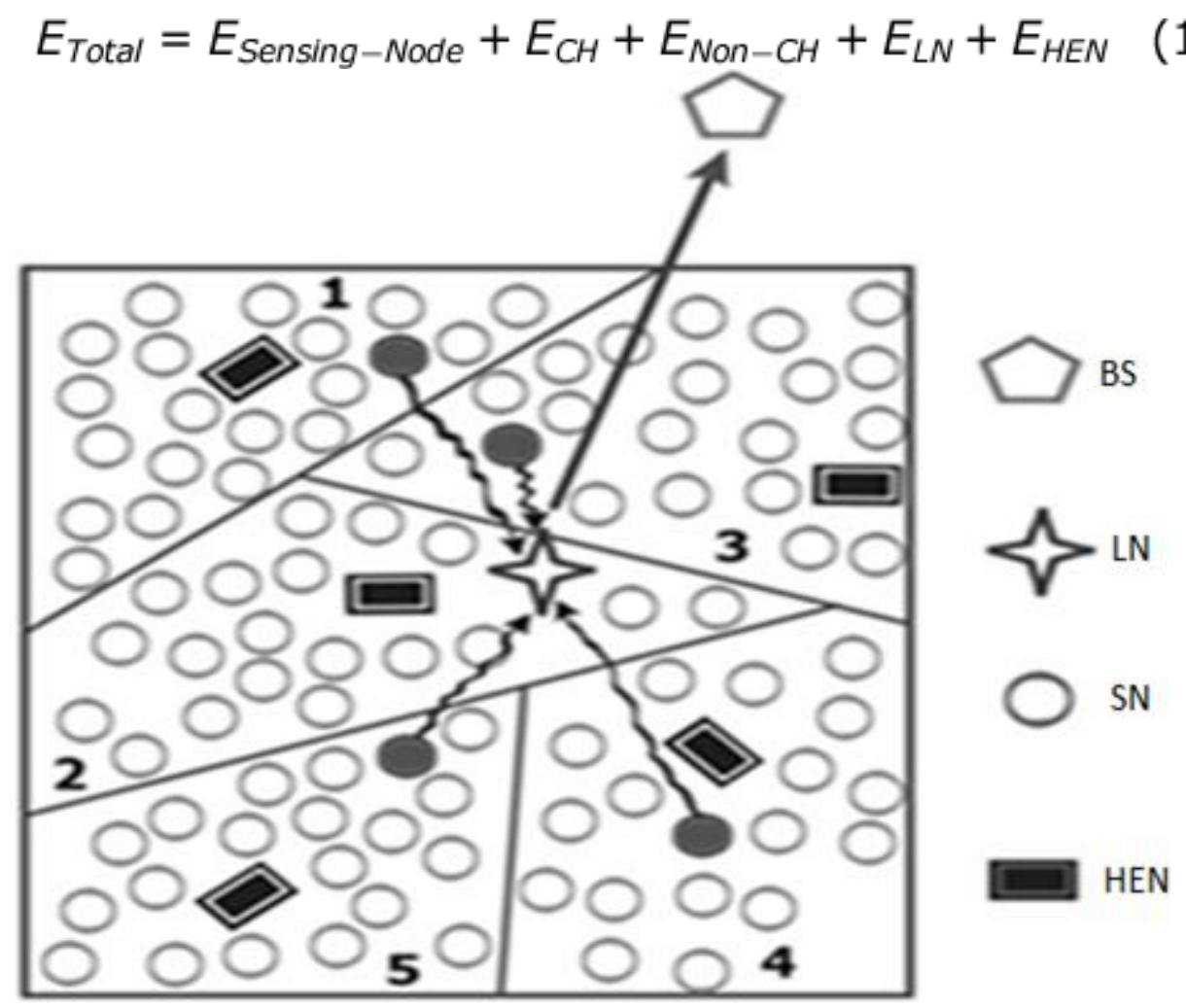

Fig.7.Steady-State Data Transfer of Our Proposed Algorithm

\section{CONCLUSION}

All Centralized Cluster-based routing protocols efficiently utilize limited energy of the nodes by rotating nodes as CHs. However, these protocols require nodes to transmit location and energy information to Base Station at the start of each round. In order to reduce the burden on sensor nodes, we proposed an algorithm in which a small percentage of High-energy nodes are used to convey each node's information to the Base Station. These nodes remain in sleep mode most of the time. In order to reduce energy load further, Cluster Head advertisement phase is eliminated as this function is performed by Base Station which has sufficient energy resources. These $\mathrm{CHs}$ gather data from their respective Clusters and transmit to a Leader node being selected by the Base Station based on its residual energy. This node transmits the final aggregated data to the Base Station.

We developed an energy model for our proposed approach and this energy model can be applied to any clustering protocol depending on the application and the choice of nodes to be used. We believe, the proposed energy model is the first of its kind in Cluster-based routing protocols. However, we have also calculated energy levels for Leader node, Sensing Node and High-Energy node to compute overall energy consumptions in a WSN.

Currently we are working on the validation and improvements on our proposed scheme using simulation models. In future, we aim to use these High-Energy nodes as $\mathrm{CHs}$ at later rounds when the energy of the sensing nodes is very low. Also as part of future work, we will investigate the behavior of our algorithm with the introduction of mobile nodes along with investigation of various security aspects and measures for our algorithm to make it more robust and secure. 


\section{REFERENCES}

[1]. Akyildiz, I.F., Su W., Sankarasubramaniam Y., Cayirci, E.: Wireless sensor networks: a survey. Elsevier Computer Networks 38, pp. 393-422 (2002)

[2]. Harrison, E.: Wireless Sensor Networks to Grow to \$2B in 2021: Report TMCNET FEATURE, August (2011)

[3]. Akkaya, K., Younis, M.: A survey on routing protocols for wireless sensor networks. Elsevier Ad Hoc Networks 3, pp.325-349 (2005)

[4]. Boyinbode, O., Le, H., Mbogho, A., Takizawa, M., Poliah, R.: A Survey on Clustering Algorithms for Wireless Sensor Networks, Proceedings of $13^{\text {th }}$ international conference on Network Based Information Systems, (2010)

[5]. Heinzelman, W. R., Chandrakasan, A., Balakrishnan, H.: An Application-Specific Protocol Architecture for Wireless Microsensor Networks. Proceedings of IEEE Transaction on Wireless Communication VOL. 1, NO. 4, pp.660-670 (2002)

[6]. Murata, T. and Ishibuchi, H.: Performance Evaluation of Genetic Algorithms for Flow shop Scheduling Problems", Proceedings of First IEEE Conference on Evolutionary Computation, volume 2, pp. 812-817 (1994)

[7]. Heinzelman, W. R., Chandrakasan, A., Balakrishnan, H.: Energy-Efficient Communication Protocol for Wireless Microsensor Networks. Proceedings of 33rd Hawaii International Conference on System Sciences (2000)

[8]. Muruganathan, S. D., Daniel C. F., Rolly, M.A., Bhasin, I., Fapojuwo, A O.: A Centralized EnergyEfficient Routing protocol for Wireless Sensor Networks, Proceedings of IEEE Radio Communications (2005)

[9]. S. Ghiasi et al., "Optimal Energy Aware Clustering in Sensor Networks," MDPI Sensors, vol. 2, no. 7, pp. 258-269 (2002)

[10]. Varma, S., Nigam, N., Tiwary, U.S.: Base Station Initiated Dynamic Routing Protocol for Heterogeneous Wireless Sensor Network using Clustering Proceedings of IEEE Wireless Communication and Sensor Networks (2008)

[11]. Kusdaryono, A., Lee, K. O.: A Clustering Protocol with Mode Selection for Wireless Sensor Network Proc. Journal of Information Processing Systems, Vol.7, No.1 (March 2011)

[12]. Heinzelman, W.R.: Application-Specific Protocol Architectures for Wireless Networks. PhD. Dissertation (2000) 